\title{
Putting the brakes on BTLA in T cell-mediated cancer immunotherapy
}

\author{
Chrystal M. Paulos and Carl H. June
}

Abramson Family Cancer Research Institute and Department of Pathology and Laboratory Medicine, University of Pennsylvania, Philadelphia, Pennsylvania, USA.

\begin{abstract}
Attenuating coinhibitory molecules for the treatment of cancer is gaining a great deal of attention as a strategy for immunotherapy. The $B$ and $T$ lymphocyte attenuator (BTLA, CD272) is a novel coinhibitory molecule structurally and functionally related to CTLA-4 and PD-1. A study in this issue of the JCI by Derré et al. reveals that BTLA is expressed on virus-specific human $\mathrm{CD8}^{+} \mathrm{T}$ cells but is progressively downregulated after their differentiation from a naive to effector phenotype (see the related article beginning on page 157). Surprisingly, tumor-specific human $\mathrm{CD8}^{+} \mathrm{T}$ cells continue to express BTLA even after their differentiation to an effector phenotype. Remarkably, vaccination of melanoma patients with CPG led to BTLA downregulation on tumor-specific human $\mathrm{CD8}^{+} \mathrm{T}$ cells, concomitant with restoration of their functionality. We discuss these findings in the context of the expanding field of cosignaling molecules and their implications for $T$ cell-based therapies for cancer.
\end{abstract}

Two families of cosignaling molecules, the CD28 family and the TNF receptor (TNFR) family, are master regulators of the immune system at the cell surface (1-3). During cellto-cell contact, specific recognition occurs between various cosignaling molecules, and this interaction ignites a plethora of signaling events, resulting in either the activation (costimulation) or the attenuation (coinhibition) of $\mathrm{T}$ cell function and proliferation (Figure 1). Indeed, cosignaling molecules are among the first responders of the immune system to self, foreign, and tumor antigens. A key feature of cosignaling molecules is that their functions are dependent on the TCR signal, and these cosignaling molecules (often referred to as "signal 2") are necessary to direct, modulate, and fine-tune the TCR signal (often referred to as "signal 1").

\section{Overview of lymphocyte cosignaling}

It has been nearly 40 years since Bretscher and Cohn first proposed the "two-signal" model for $\mathrm{T}$ cell activation, and while the details have become increasingly complex, the simplicity of the model provides a basic framework with which to understand mechanisms that maintain immune tolerance (4). In the mid-1980s, CD28 was identified

Conflict of interest: The authors have declared that no conflict of interest exists.

Citation for this article: J. Clin. Invest. 120:76-80 (2010). doi:10.1172/JCI41811. as the first cosignaling molecule. CD28 provides the dominant signals required for full activation of naive lymphocytes and thus is called a costimulatory molecule. Shortly after the discovery of CD28, cytotoxic T lymphocyte antigen-4 (CTLA-4) was identified as a protein that shares ligands and structural homology with CD28 (5). In contrast to CD28, however, CTLA-4 was found to inhibit $\mathrm{T}$ cell responsiveness and is thus considered a coinhibitory molecule. Manipulation of the CTLA-4 pathway using antibody blockade has shown considerable promise for the treatment of patients with cancer, and these clinical data have motivated investigators to search for other coinhibitory molecules for clinical benefit.

Several additional coinhibitory molecules have been identified in the past decade, including programmed death-1 (PD-1), lymphocyte activation-gene 3 (LAG-3), CD160, and the B and T lymphocyte attenuator (BTLA). BTLA is the most recently identified receptor of the $\mathrm{CD} 28$ family and is structurally related to CTLA-4 and PD-1 (6). BTLA binds the herpes virus entry mediator (HVEM). Interestingly, HVEM is a member of the TNFR family; and its interaction with BTLA is the first demonstration of crosstalk between the CD28 and TNFR families (7), an observation that has profound implications for the complexity of regulation of the innate and acquired immune systems.
In contrast to mice lacking CTLA-4 or PD-1, young BTLA-deficient mice show no obvious signs of autoimmunity; however, with age, they develop autoimmune hepatitis and other signs of immunopathology (8). Furthermore, mice lacking BTLA are far more susceptible to EAE, a model of $\mathrm{T}$ cellmediated autoimmune disease that shares several features with MS (6). Interestingly, BTLA blockade prevents proliferation and cytokine production by T cells, while BTLA triggering leads to decreased antimicrobial and autoimmune responses in mice, suggesting that BTLA may have an important role in restraining cellular immunity. However in humans, little is known about the contribution of BTLA to tolerance and immunopathology, or to its functional effect on antigen-specific $\mathrm{T}$ cells in vivo.

\section{Cancer patients have abnormal expression of BTLA}

In this issue of the JCI, Derre and coworkers report that naive human $\mathrm{CD} 8^{+} \mathrm{T}$ cells express high levels of BTLA on their cell surface (9). However in bulk and influenzaspecific $\mathrm{CD}^{+} \mathrm{T}$ cells in healthy donors and cancer patients, they found that the surface expression of BTLA is gradually downregulated during differentiation of human $\mathrm{CD}^{+}$ $T$ cells from the naive to effector cell phenotype (Figure 2A). Remarkably, this is not the case for tumor-specific human $\mathrm{CD} 8^{+} \mathrm{T}$ cells. In sharp contrast, these cells persistently expressed high levels of BTLA in vivo and remained susceptible to functional inhibition by the BTLA ligand HVEM (Figure 2B). Importantly, the authors demonstrate abundant expression of HVEM in a subset of melanomas and show that tumor-specific $\mathrm{CD}^{+} \mathrm{T}$ cells in these patients were inhibited by interaction of BTLA with HVEM on the tumor, even after progressive differentiation of the $T$ cells.

Additional investigation by Derré et al. revealed persistent BTLA expression on spontaneously elicited tumor antigen Melan- $\mathrm{A}^{\text {Mart-1}}-$ specific $\mathrm{CD}^{+} \mathrm{T}$ cells from melanoma patients and after conven- 


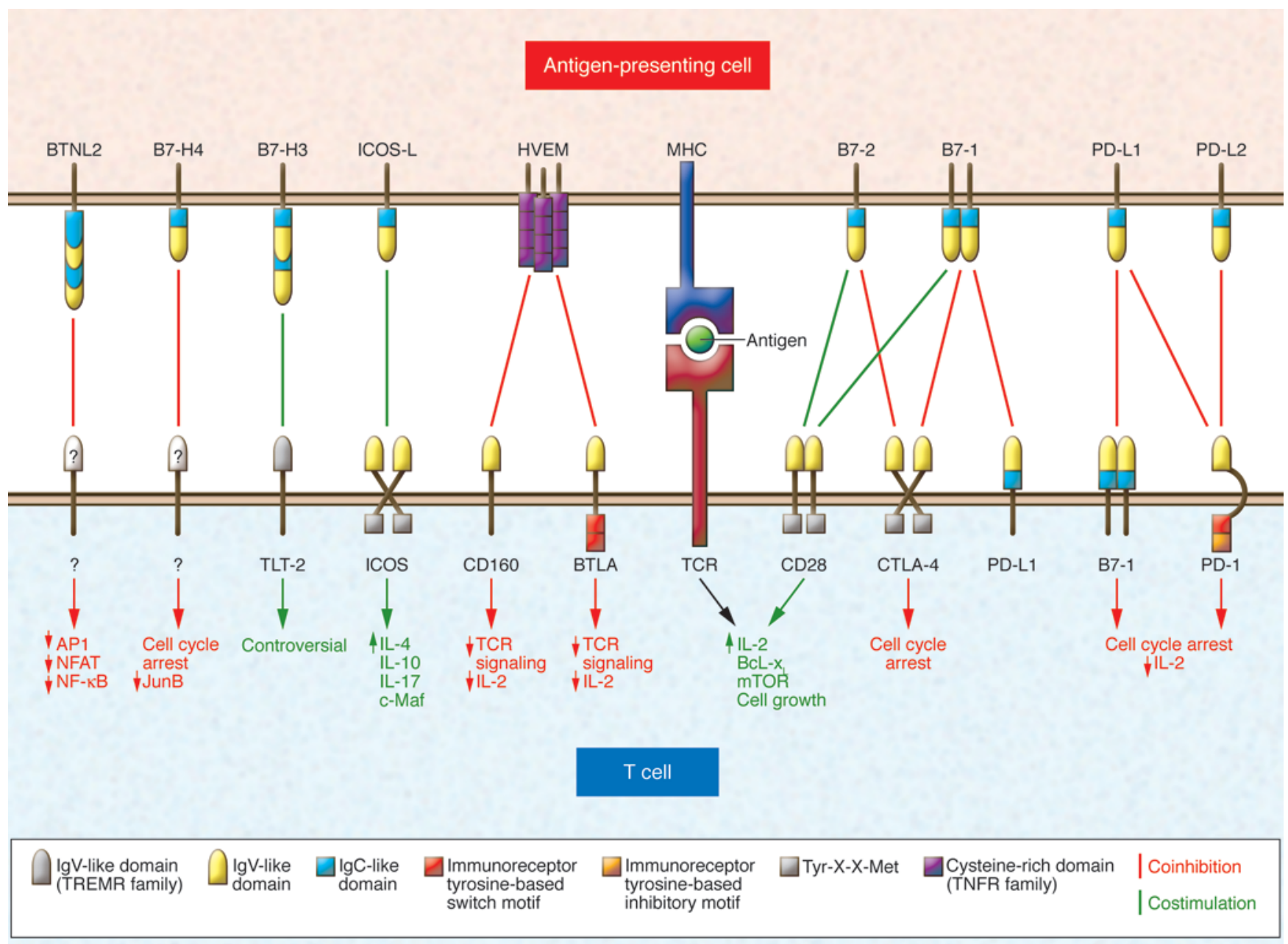

\section{Figure 1}

The B7/CD28 family interactions and their function outcomes. The B7/CD28 family members are characterized by their common immunologlobulin extracellular binding domains, and their signaling pathways are driven mainly by their intracellular, tyrosine-containing motifs. In addition to the specific antigen-dependent signal provided by the MHC-antigen complex to the TCR, the cell-surface interactions involving the B7/CD28 family of cosignaling molecules provide a second signal to T cells to enhance or inhibit the TCR signal, which influences the proliferative capacity and functional fate of the T cell. In addition to CD28 ligation by B7-1 and B7-2, T cell costimulation can be mediated by ligation of inducible costimulator (ICOS) via its ligand ICOS-L or can be mediated by ligation of the newly reported trim-like transcript 2 (TLT-2) via its ligand B7-H3. Also, in addition to CTLA-4, BTLA, CD160, and PD-1, which exert their effects via ligation by their respective ligands, is a new molecule referred to as butyrophilin/B7-like molecule (BTNL2), a coinhibitory molecule that mediates signaling of activator protein 1 (AP1) and nuclear factor of activated T cells (NFAT) via a ligand that remains elusive (as indicated by a question mark). Costimulatory interactions are shown in green, coinhibitory interactions in red. TREMR, triggering receptors expressed on myeloid cells receptor. Adapted with permission from Cell (21).

tional peptide vaccination consisting of a Melan- $\mathrm{A}_{26-35}$ peptide and incomplete Freund's adjuvant (9). As modeled in Figure $2 \mathrm{~B}$, these cells possess minimal functionality upon tumor encounter. Surprisingly, the addition of a TLR9 agonist, CPG, to the vaccine comprising Melan- $\mathrm{A}_{26-35}$ peptide and incomplete Freund's adjuvant led to "normalization": progressive BTLA downregulation in vivo on those cells and resistance to BTLA-HVEM-mediated inhibition, as assessed by the capacity of these cells to produce IFN- $\gamma$ after recognition of tumor antigen (Figure 2C).
This work (9) catapults the field of tumor immunotherapy forward by demonstrating, for the first time to our knowledge, that BTLA is a valid target for cancer immunotherapy. The coinhibitory molecule BTLA can inhibit tumor-specific human $\mathrm{CD}^{+} \mathrm{T}$ cells; and vaccination with CPG adjuvants, at least in part, overcomes this barrier by downregulating BTLA. CPG-mediated downregulation of BTLA correlates with restoration of the in vivo effector function of tumor-specific human $\mathrm{CD}^{+} \mathrm{T}$ cells. These data underscore the therapeutic potential of exploiting the BTLA pathway to treat patients with cancer and infectious disease as well as patients with autoimmunity. Thus, a therapeutic immune intervention to treat infectious or malignant disease would involve blocking BTLA-mediated $\mathrm{T}$ cell inhibition, whereas this pathway could be pharmacologically augmented to promote BTLA-mediated T cell inhibition or tolerance for patients with autoimmune diseases or for allograft recipients.

\section{Exciting findings lead to new questions}

The current studies raise fundamental questions for further investigation. The present 


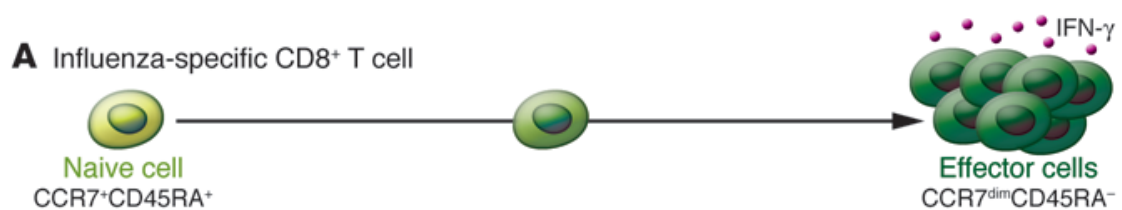

Gain of function

BTLA level Differentiation

B Tumor Melan-A $\mathrm{A}^{\text {Mart-1}}-$ specific $\mathrm{CD} 8^{+} \mathrm{T}$ cell

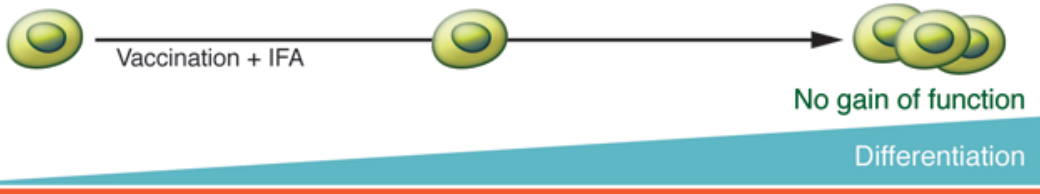

BTLA level

\section{Tumor Melan- $\mathrm{A}^{\text {Mart-1}}-$ specific $\mathrm{CD}^{+} \mathrm{T}$ cell plus $\mathrm{CpG}$ vaccination} Vaccination + IFA + CpG
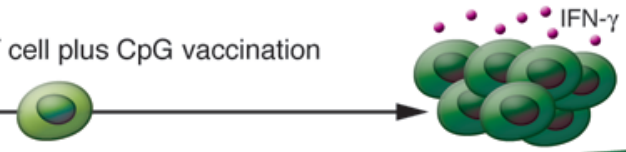

Gain of function

\section{BTLA level}

Differentiation

\section{Figure 2}

BTLA expression inhibits tumor-specific human $\mathrm{CD} 8^{+} \mathrm{T}$ cell function, which can be overcome by vaccination with $\mathrm{CpG}$. In this issue of the $\mathrm{JCI}$, Derré et al. report that naive human CD8+ $T$ cells express high levels of the coinhibitory molecule BTLA on their surface. They find that BTLA can inhibit the function of tumor-specific human $\mathrm{CD}^{+} \mathrm{T}$ cells. In vivo, vaccination of melanoma patients with $\mathrm{CpG}$ dampened this inhibition, at least in part, by downregulating BTLA. (A) The surface expression of BTLA is gradually downregulated during differentiation of virus-specific human $C D 8^{+} T$ cells from a naive $\left(C C R 7{ }^{+} C D 45 R A^{+}\right)$to an effector cell phenotype (CCR7 ${ }^{\text {dim }}$ CD45RA ${ }^{-}$). These differentiated cells produce high amounts of IFN- $\gamma$. (B) The surface expression of BTLA is maintained on tumor Melan-AMart-1-specific human CD8 ${ }^{+}$T cells from patients vaccinated with conventional peptide vaccination consisting of a Melan- $A_{26-35}$ peptide and incomplete Freund's adjuvant (IFA) even after their differentiation to an effector phenotype, and this is associated with impaired functionality, as indicated by their reduced capacity to produce IFN- $\gamma$. (C) The vaccination of melanoma patients with the TLR agonist

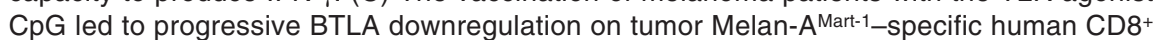
T cells and resistance to BTLA-HVEM-mediated functional inhibition and robust production of IFN- $\gamma$. These data not only underscore the therapeutic potential of $\mathrm{CpG}$ but also reveal the clinical importance of the BTLA pathway.

data convincingly demonstrate that vaccination with CPG downregulates BTLA expression on tumor-specific T cells in patients in vivo and that this somehow results in the enhanced activation and function of these cells (9). However, at a clinical level, it will be important to confirm these data in future studies involving randomized cohorts of patients in order to exclude seemingly minor differences in the performance status and other variables of patients enrolled in cancer vaccine studies that can have major impact on the observed responses. Moreover, further studies will be required to obtain a mechanistic understanding of this effect. For example, Derré and colleagues have not yet examined how this vaccination strategy might impact the expression of CD160, a coinhibitory molecule present on the same patient cells. HVEM interacts not only with BTLA but also with CD160 and LIGHT (an inducible lymphotoxin-like molecule that competes for HVEM) (Figure 3). While ligation of LIGHT via HVEM-expressing tumors stimulates $T$ cell responses (Figure 3C), ligation of BTLA (and perhaps CD160) via HVEM-expressing tumors transmits coinhibitory signals to $\mathrm{T}$ cells (Figure 3, A and B). Thus, HVEM is a bidirectional molecular switch capable of regulating $\mathrm{T}$ cell activation by costimulation or coinhibition, the outcome of which depends on the ligand engaged. It is conceivable that vaccination also downregulates the expression of CD160 and that this also augments $\mathrm{T}$ cell activation. Indeed, the HVEMBTLA interaction is likely to be exceedingly complex, as T cells can express 5 different ligands for HVEM (10), and therefore the interactions within this receptor system require further investigation. Such investigations will be important, as they may shed light onto whether the CD160 pathway is of therapeutic merit as well, or if the molecule is simply a passenger with BTLA.

Speiser and colleagues previously reported efforts to use TLR agonists in conjunction with vaccination in patients with melanoma (11). Combining the TLR9 agonist CpG with a Melan- $\mathrm{A}_{26-35^{\text {MART-1 }}}$ peptide and incomplete Freund's adjuvant increased the number of Melan- $\mathrm{A}_{26-35^{\mathrm{MART}}-1 \text {-specific } \mathrm{T}}$ cells by greater than 10 -fold compared with vaccination without $\mathrm{CPG}$. This heightened the immune response; however, it did not promote tumor regression. These findings might imply that the Melan- $\mathrm{A}_{26-35^{\mathrm{MART}}-{ }_{-}}$ specific $T$ cells induced by $C p G$ and vaccination are functionally tolerized. For example, the tumor-reactive T cells might be tolerized by Tregs, as hypothesized by this group and others, who reported an elevation of Tregs in the tumors of vaccinated patients receiving CpG. The present work implies that it is not only Tregs that hamper the immunity in these patients. Besides Tregs, inhibition via BTLA and perhaps CD160 may play a key role, given that BTLA may be expressed on virtually all tumor-specific $\mathrm{CD}^{+} \mathrm{T}$ cells at every stage of differentiation. Discerning how CpG differentially impacts on tumorspecific $\mathrm{T}$ cell effector functions by Tregs versus BTLA will be important.

It is intriguing that BTLA expression is maintained at high levels despite tumorspecific $\mathrm{CD}^{+} \mathrm{T}$ cell differentiation to effector cells (9), unlike the case of influenza virus-specific $\mathrm{CD}^{+} \mathrm{T}$ cells, which progressively downregulate their BTLA expression after differentiation from a naive to effector phenotype. Interestingly, however, significant proportions of EBV- and CMV-specific $\mathrm{CD}^{+} \mathrm{T}$ cells persistently expressed BTLA, in contrast to influenza-specific $\mathrm{CD}^{+} \mathrm{T}$ cells, suggesting that either differences in TCR affinity or chronic antigenic exposure may sustain BTLA expression and thus inhibi- 


\section{Tolerance}

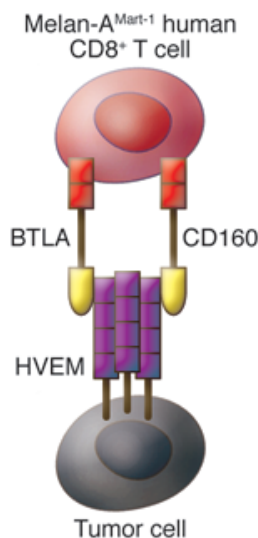

Coinhibition of

T cell response
Tolerance

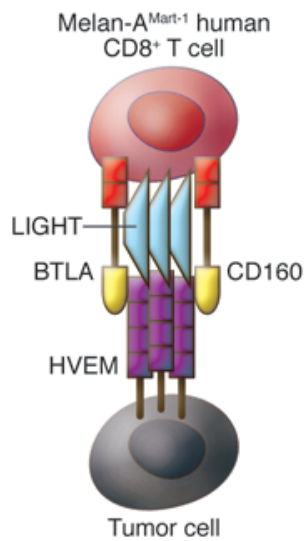

Coinhibition of T cell response
Immunity

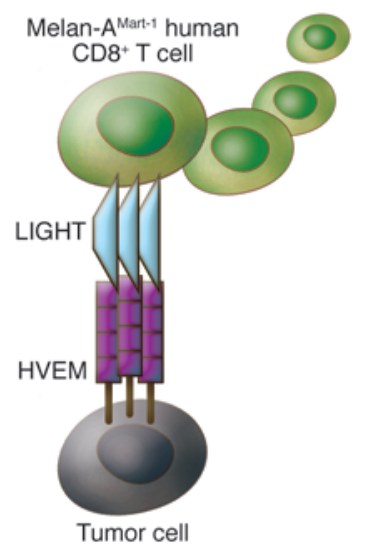

Costimulation of T cell response

\section{Figure 3}

Models of interaction among HVEM, BTLA, CD160, and LIGHT and their various functional effects on tumor-specific human CD8 ${ }^{+}$T cells. BTLA, CD160, and LIGHT are differentially expressed on tumor-specific CD8 ${ }^{+} \mathrm{T}$ cells, and depending on their expression, they can mediate distinct outcomes: immune tolerance or effective immunity against tumor targets. Three potential interactions are shown. Left: If BTLA or CD160 is expressed and LIGHT expression is either low or absent, the coinhibitory BTLA-CD160-HVEM complex will be dominant, resulting in negative regulation of the tumor-specific $C D 8^{+} \mathrm{T}$ cell by the human tumor. Middle: If LIGHT, BTLA, and CD160 are all expressed, they might form a complex with HVEM. This could trimerize HVEM, resulting in positive or negative regulation of the T cell by the tumor. Right: If LIGHT is expressed with little to no BTLA or CD160, the tumor-specific T cells receive a positive signal from the HVEM-expressing tumor, resulting in robust functional activation of the tumor-specific $T$ cell. Thus, attenuation of BTLA via either CpG or antibody blockade might augment $T$ cell-mediated immunotherapy for cancer. Potent tumor-specific $T$ cell responses are mediated with conventional vaccination and $\mathrm{CpG}$, which downregulates BLTA expression on T cells, as revealed by new findings reported by Derré et al. (9) and as represented in the right panel. Adapted with permission from Trends in immunology (22).

tion of specific $\mathrm{CD}^{+} \mathrm{T}$ cells. Understanding the mechanism by which some antigenspecific T cells downregulate BTLA while others do not will be important.

\section{Future directions}

Given that $\mathrm{CpG}$ triggers innate immunity via TLR9, the question naturally arises, Do all forms of innate activation downregulate BTLA expression, and does this process enhance tumor-specific $T$ cell activation? More than 100 years ago, William Coley tried to exploit the innate arm to treat patients with cancer $(12)$. He reported tumor regression in some of his patients given the mixture of bacteria consisting of heat-killed cultures of Streptococci and Serratia marcescens. Since then, investigators have made major advancements in safely triggering innate immunity (13). Furthermore, it is now accepted that lymphodepletion with chemotherapy or total body irradiation activates the innate immune system via release of TLR4 agonists not only from the tumor but also from the radiation-injured gut $(14,15)$. Thus, it will be interesting to determine how innate activators, such as TLR agonists or chemotherapy, impact on the expression of BTLA as well as on other coinhibitory and costimulatory molecules expressed on tumor-specific T cells.

Human B cells highly express TLR9, and given that melanoma patients have recently been shown to have major abnormalities in the $\mathrm{B}$ cell compartment (16), it is possible that perturbed availability of B cells with abundant TLR9 contributes to the persistent BTLA expression in the self-reactive $\mathrm{T}$ cells described in cancer patients.

These novel findings in patients with cancer support the need to develop therapeutic interventions to interfere with BTLA-mediated immune regulation. Tumor-specific $\mathrm{T}$ cells are often primed on "bad" or immature APCs, and one view has been that this leads to $\mathrm{T}$ cells with a poor proliferative capac- ity and impaired functionality. The present results showing restoration of seemingly normal $\mathrm{T}$ cell differentiation and at least partial reversal of function with BTLA antagonism are a source of optimism that suboptimal antitumor responses can be rescued in cancer patients (9). Furthermore, given the initial promising clinical results to date of the adoptive transfer of $T$ cells in patients with melanoma, the stage is now set to incorporate $\mathrm{CpG}$ adjuvanted vaccination strategies with BTLA blockade therapy. Importantly, this work has broader implications, similar to those revealed with the discovery of CTLA-4. Given that BTLA is expressed on cells with a naive phenotype, perhaps its therapeutic disruption will yield greater promise for the treatment of patients with cancer than that achieved with CTLA-4 blockade, or perhaps their combined antagonism will permit optimal $\mathrm{T}$ cell-mediated tumor immunity.

From a therapeutic perspective, there is a strong rationale for developing antagonistic and perhaps agonistic antibodies to BTLA; however, the complexity of this receptor and ligand system may present a challenge. Furthermore, since antagonism of both CTLA-4 and PD-1 has already shown promise in clinical trials (17), it is likely that combination blockade may be more potent and more toxic, given that the mechanism of action of each of these coinhibitory receptors is different $(3,10)$.

Another issue to be resolved is clarifying the mechanism of CpG-mediated "correction" of BTLA expression. It is possible that the CPG adjuvant results in TLR9-mediated cross-presentation on plasmacytoid dendritic cells and that this remedies the originally suboptimal presentation that occurs on melanoma cells via direct presentation or on immature, myeloid-derived dendritic cells during the natural course of tumor progression. Another possibility to be excluded is whether CPG may directly increase the immunogenicity of melanoma cells.

The present results suggest that in situations of chronic antigen exposure, such as cancer and EBV and CMV infection, BTLA expression is sustained, while in situations where antigen persistence is extinguished, BTLA expression is turned off. It may be that contexts that promote the establishment of true memory require downregulation of BTLA. Recent studies in mice indicate that BTLA is required for the induction of tolerance (18), and it is perhaps not surprising that tumors may take advantage of this pathway to inhibit adaptive immunity. 
Finally, recent results indicate that the induction of expression of ICOS, a costimulatory molecule related to $\mathrm{CD} 28$, on $\mathrm{CD}_{4}{ }^{+}$ cells correlates with positive responses to cancer immunotherapy in patients with prostate cancer $(19,20)$. The present results (9) suggest that persistent BTLA expression on the surface of $\mathrm{T}$ cells with tumor specificity is a poor prognostic biomarker and that the loss of BTLA expression consequent to cancer immunotherapy may be a positive biomarker of response. The data reported in this issue of the JCI by Derré and coworkers are robust and are likely to provide insight particularly given that they are derived from human studies, which often reveal disease mechanisms not evident in murine studies.

\section{Acknowledgments}

We thank the scientific and clinical team and the patients at the University of Pennsylvania for help and guidance in the development of new cancer immunotherapies. This work was supported in part by NIH grant 5R01CA105216 to C.H. June and NIH training grant support for C.M. Paulos.

Address correspondence to: Chrystal M. Paulos or Carl H. June, Abramson Family Cancer Research Institute and Department of Pathology and Laboratory Medicine, University of Pennsylvania, BRB II/III, Room 554,
421 Curie Boulevard, Philadelphia, Pennsylvania 19104-6160, USA. Phone: (202) 5571868 (C.M. Paulos) and (215) 573-5745 (C.H. June); Fax: (215) 573-8590; E-mail: chrystal. paulos@gmail.com (C.M. Paulos) or cjune@ exchange.upenn.edu (C.H. June).

1. Chen L. Co-inhibitory molecules of the B7-CD28 family in the control of T-cell immunity. Nat Rev Immunol. 2004;4(5):336-347.

2. Greenwald RJ, Freeman GJ, Sharpe AH. The B7 family revisited. Annu Rev Immunol. 2005;23:515-548.

3. Riley JL, June CH. The CD28 family: a T-cell rheostat for therapeutic control of T-cell activation. Blood. 2005;105(1):13-21.

4. Bretscher P, Cohn M. A theory of self-nonself discrimination. Science. 1970;169(950):1042-1049.

5. Brunet JF, et al. A new member of the immunoglobulin superfamily - CTLA-4. Nature. 1987;328(6127):267-270.

6. Watanabe $\mathrm{N}$, et al. BTLA is a lymphocyte inhibitory receptor with similarities to CTLA-4 and PD-1. Nat Immunol. 2003;4(7):670-679.

7. Sedy JR, et al. B and T lymphocyte attenuator regulates $T$ cell activation through interaction with herpesvirus entry mediator. Nat Immunol. 2005;6(1):90-98.

8. Oya Y, et al. Development of autoimmune hepatitis-like disease and production of autoantibodies to nuclear antigens in mice lacking $B$ and $T$ lymphocyte attenuator. Arthritis Rheum. 2008;58(8):2498-2510.

9. Derré L, et al. BTLA mediates inhibition of human tumor-specific $\mathrm{CD}^{+} \mathrm{T}$ cells that can be partially reversed by vaccination. J Clin Invest. 2010;120(1):157-167.

10. Cai G, Freeman GJ. The CD160, BTLA, LIGHT/ HVEM pathway: a bidirectional switch regulating Tcell activation. Immunol Rev. 2009;229(1):244-258.

11. Speiser DE, et al. Rapid and strong human CD8+ $\mathrm{T}$ cell responses to vaccination with peptide, IFA, and CpG oligodeoxynucleotide 7909. J Clin Invest.
2005;115(3):739-746

12. Coley WB. The treatment of malignant tumors by repeated inoculations of erysipelas. With a report of ten original cases. 1893. Clin Orthop Relat Res. 1991;(262):3-11.

13. Paulos CM, et al. Toll-like receptors in tumor immunotherapy. Clin Cancer Res. 2007;13(18 pt 1):5280-5289.

14. Paulos CM, et al. Microbial translocation augments the function of adoptively transferred self/tumorspecific CD8+ T cells via TLR4 signaling. J Clin Invest. 2007;117(8):2197-2204.

15. Apetoh L, et al. Toll-like receptor 4-dependent contribution of the immune system to anticancer chemotherapy and radiotherapy. Nat Med. 2007;13(9):1050-1059.

16. Carpenter EL, et al. Collapse of the CD27+ B-cell compartment associated with systemic plasmacytosis in patients with advanced melanoma and other cancers. Clin Cancer Res. 2009;15(13):4277-4287.

17. Melero I, Martinez-Forero I, Dubrot J, Suarez N, Palazón A, Chen L. Palettes of vaccines and immunostimulatory monoclonal antibodies for combination. Clin Cancer Res. 2009;15(5):1507-1509.

18. Liu X, et al. Cutting edge: a critical role of $B$ and $T$ lymphocyte attenuator in peripheral $\mathrm{T}$ cell tolerance induction. J Immunol. 2009;182(8):4516-4520.

19. Liakou CI, et al. CTLA-4 blockade increases IFNgamma-producing CD4+ICOShi cells to shift the ratio of effector to regulatory $\mathrm{T}$ cells in cancer patients. Proc Natl Acad Sci U S A. 2008;105(39):14987-14992.

20. Chen $\mathrm{H}$, et al. Anti-CTLA-4 therapy results in higher CD4+ICOShi T cell frequency and IFNgamma levels in both nonmalignant and malignant prostate tissues. Proc Natl Acad Sci U S A. 2009;106(8):2729-2734.

21. Paterson A, Vanuri V, Sharpe A. SnapShot: B7/ CD28 costimulation. Cell. 2009;137(5):974-975.

22. Croft M. The evolving crosstalk between co-stimulatory and co-inhibitory receptors: HVEM-BTLA. Trends Immunol. 2005;26(6):292-294.

\title{
Sodium channels gone wild: resurgent current from neuronal and muscle channelopathies
}

\author{
Stephen C. Cannon ${ }^{1}$ and Bruce P. Bean² \\ 1Department of Neurology, University of Texas Southwestern Medical Center, Dallas, Texas, USA. \\ 2Department of Neurobiology, Harvard Medical School, Boston, Massachusetts, USA.
}

\begin{abstract}
Voltage-dependent sodium channels are the central players in the excitability of neurons, cardiac muscle, and skeletal muscle. Hundreds of mutations in sodium channels have been associated with human disease, particularly genetic forms of epilepsy, arrhythmias, myotonia, and periodic paralysis. In this issue of the JCI, Jarecki and colleagues present evidence suggesting that many such mutations alter the gating of sodium channels to produce resurgent sodium current, an unusual form of gating in which sodium channels reopen following an action potential, thus promoting the firing of another action potential (see the related article beginning on page 369). The results of this study suggest a widespread pathophysiological role for this mechanism, previously described to occur normally in only a few types of neurons.
\end{abstract}

Conflict of interest: The authors have declared that no conflict of interest exists.

Citation for this article: J. Clin. Invest. 120:80-83 (2010). doi:10.1172/JCI41340.
Our understanding of channelopathies human disorders arising from mutations of ion channel genes - has gone through several waves of discovery. First, there was the implication that ion channels may play a causal role in disease pathology from the observation of abnormal ionic conductances in muscle biopsied from individuals with myotonia or periodic paralysis, studied using microelectrode recording $(1,2)$. Then came identification of mutations in ion channel genes, made possible by discovery of ion channel gene superfamilies; disease-associated mutations were identified by genome-wide linkage studies or by a candidate gene approach guided by the discovery of aberrant conductances in affected cells (3). This approach enabled the identification of numerous channelopathies in heart, 